\title{
APPLICATION OF VECTOR FITTING TO STATE EQUATION REPRESENTATION OF TRANSFORMERS FOR SIMULATION OF ELECTROMAGNETIC TRANSIENTS
}

\author{
Bjørn Gustavsen* \\ Adam Semlyen \\ Department of Electrical and Computer Engineering \\ University of Toronto \\ Toronto, Ontario, Canada M5S 3G4
}

* On leave from the Norwegian Electric Power Research Institute (EFI), Trondheim, Norway.

\begin{abstract}
This paper presents an efficient methodology for transient modeling of power transformers based on measured or calculated frequency responses. The responses are approximated with rational functions using the method of vector fitting. The resulting model is dynamically stable since only negative poles are used, and input-output stability is assured by a novel adjusting procedure. The methodology is used to formulate two different transformer models: 1) a full phase domain model realized column-by-column, and 2) a model utilizing modal diagonalization. The application of the models is demonstrated for a 410 MVA generator step up transformer.
\end{abstract}

\section{INTRODUCTION}

The transient behavior of power transformers is very difficult to model accurately. The current/voltage characteristics at the terminals are strongly frequency dependent, and usually involve resonance phenomena. As this behavior cannot be deduced from the power frequency data provided by the manufacturer, one has to acquire the needed frequency responses either from detailed calculations based on internal geometry, or from terminal measurements. Such frequency responses constitute the basis for model formulation.

The frequency responses can be included in the time domain calculations by means of numerical convolution, but the resulting model would be computationally very time consuming. Therefore, one will normally seek to replace the original frequency responses by rational function approximations, as this enables a more efficient time domain implementation. Rational function approximations can in principle be calculated by fitting rational polynomials to the data [1]. However, the inherent ill-conditioning limits this method to low order approximations. Also, some poles of the resulting fit may turn out to be unstable. A way of overcoming these problems is by iteratively fitting partial fractions to the data [2]. This methodology is complicated in the sense that the frequency responses must be scrutinized in advance to decide how many and which type of fractions to use (real or complex conjugate pairs). Also, the success of the method is dependent on the starting values used in the iteration.

A sufficient requirement for the resulting transformer model to give a stable simulation is that all of its poles lie in the left half plane (dynamic stability), and that all eigenvalues of the resulting admittance matrix lie in the right half plane (input-output stability). Many of the proposed fitting procedures fulfill the first requirement, but the last one can in practice only be assessed after the fitting has been carried out. The problem of input-output stability is highly relevant in the case of transformers, as the internal losses are very small. Thus, even a quite accurately fitted

PE-673-PWRD-0-03-1997 A paper recommended and approved by the IEEE Transmission and Distribution Committee of the IEEE Power Engineering Society for publication in the IEEE Transactions on Power Delivery. Manuscript submitted October 18, 1996; made available for printing March 26, 1997. model can give unstable simulation results, depending on the connected network.

In this paper we show that the recently developed method of vector fitting [3] is well suited for finding the needed rational function approximations. This method produces a state equation realization (SER) by solving an overdetermined set of equations as a least squares problem. There is no need for predefining partial fractions, and there are inherent limitations only if a fit of very high order is desired for frequency responses with many peaks. We use vector fitting to formulate two transformer models based on the short circuit admittance matrix $Y$ :

1. Fitting $Y$ column-by-column in the phase domain

2. Applying modal decomposition to $3 \times 3$ blocks of $Y$

Dynamic stability is achieved by forcing all poles to lie in the left half plane. Input-output stability is ensured by adding a correction to the resulting fit. The correction is established by examining the eigenvalues of the fitted $Y$ as function of frequency.

The methodology is demonstrated for a 410 MVA twowinding star-delta connected transformer.

In this work we neglect any nonlinear phenomena, such as saturation effects and hysteresis that may have significance at lower frequencies. If nonlinear effects need to be taken into account they have to be added on externally to the model as in [2].

\section{METHODOLOGY}

\subsection{Model formulation}

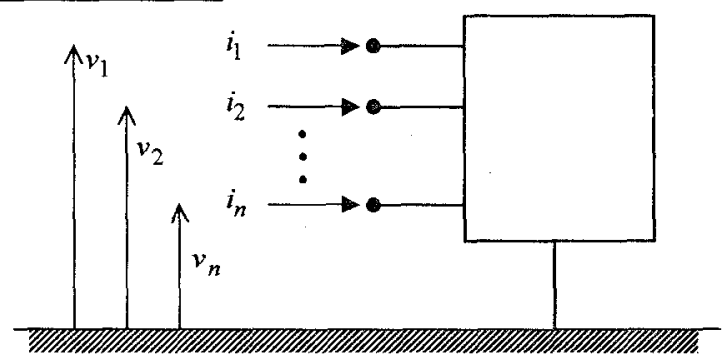

Fig. 1 n-terminal transformer

A transformer will in the following be considered as an $n$-terminal linear component, as shown in figure 1. The current/voltage relations at the terminals can in the frequency domain be expressed by the admittance matrix $Y$ :

$$
i=Y v
$$

where $i$ and $v$ are vectors of length $n$, and $Y$ is an $n \times n$ complex matrix. It follows from (1) that the columns of $Y$ can be measured one-by-one by successively applying a sinusoidal voltage to one of the terminals, with the other terminals connected to ground.

A transformer model could be obtained by representing each element of $Y$ with a state equation realization (SER). However, the time domain simulations would be somewhat tedious as this 
model involves $n^{2}$ SERs. The computational burden can be reduced by a factor of about 2 by fitting $Y$ columnwise using the same set of poles in each column [3]. This can easily be done using the method of vector fitting adopted in this paper.

In principle, one could increase the computational speed even more by applying modal diagonalization to $Y$, assuming a constant, real transformation matrix. However, this would lead to inaccurate results as the transformation matrix is in general both frequency dependent and complex.

Morched et al. [2] proposed to subdivide $Y$ into $3 \times 3$ blocks, one for each transformer winding. For a two-winding transformer, equation (1) can be written

$$
\left[\begin{array}{l}
i_{1} \\
i_{2}
\end{array}\right]=\left[\begin{array}{ll}
Y_{11} & Y_{12} \\
Y_{21} & Y_{22}
\end{array}\right]\left[\begin{array}{l}
v_{1} \\
v_{2}
\end{array}\right]
$$

where subscripts 1 and 2 denote winding 1 and 2 , respectively. Then they averaged the diagonal and off-diagonal elements within each block, thus making each block a balanced matrix of the form

$$
\left[\begin{array}{lll}
a & b & b \\
b & a & b \\
b & b & a
\end{array}\right]
$$

This allowed them to apply modal decomposition to each block, using a constant, real transformation matrix.

However, the use of balanced submatrices is not always acceptable : three-legged transformers may exhibit symmetry only about the middle leg, and transformers utilizing a delta or zigzag winding will have off-diagonal blocks with cyclic symmetry. For instance, for the star-delta connected transformer investigated in this work we found $Y$ to have the following symmetry properties:

$$
Y_{11} \approx\left[\begin{array}{lll}
a & b & c \\
b & a^{\prime} & b \\
c & b & a
\end{array}\right], Y_{21}=Y_{12} \approx\left[\begin{array}{lll}
e & f & 0 \\
0 & e & f \\
f & 0 & e
\end{array}\right], Y_{22} \approx\left[\begin{array}{lll}
g & h & h \\
h & g & h \\
h & h & g
\end{array}\right]
$$

where 1 and 2 denote the star and delta winding, respectively.

The problem of cyclic off-diagonal blocks can be overcome by using Fortescue transformation ( +-0 components). This transformation will perfectly diagonalize cyclic blocks and approximately diagonalize blocks with mid-leg symmetry. Therefore, the model will also work if there is no star-delta or zigzag connection.

Diagonal blocks are never cyclic, but may have a significant mid-leg symmetry. An analysis (see Appendix A) has shown that increased accuracy is achieved for such blocks when using $\alpha \beta 0$ transformation, instead of the prebalancing procedure used in [2].

Thus, we propose the following two transformer models :

1. Columnwise realization of $Y$ (phase domain)

2. Blockwise diagonalization of $Y$ without prebalancing :

- apply $\alpha \beta 0$ components to diagonal blocks

- apply +-0 components to off-diagonal blocks.

For a two-winding transformer, application of model 2 can be illustrated by the flow chart in figure 2 , where superscript $\mathrm{m}$ denotes modal quantities.

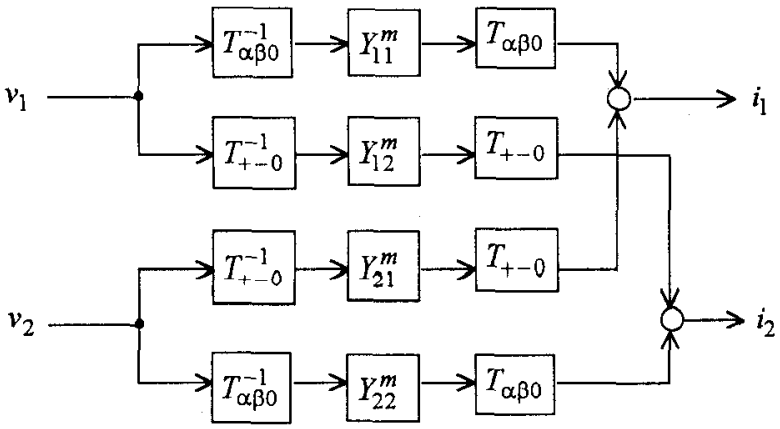

Fig. 2 Implementation of model 2 (two-winding transformer)

\subsection{Rational function approximation}

In model 1 we approximate each column $Y_{j}$ of $Y$ by a finite order state equation realization (SER)

$$
Y_{j} \approx C(s I-A)^{-1} B+D
$$

calculated using the method of vector fitting developed in [3]. Similarly, in model 2 we use vector fitting to find a SER for each diagonal element in the blocks $Y_{i j}^{m}$.

As described in [3], the fitting process involves two stages :

1. Calculation of poles ( $A$-matrix) from "starting poles"

2. Calculation of $C$ with known poles ( $B$ is normalized and fixed)

As starting poles we use real, negative poles that are linearly distributed over the frequency range where the frequency responses are to be fitted. The preference here for linearly spaced poles over logarithmically spaced poles as used in [3] is due to the responses appearing smoother when plotted on a linear frequency base. The new set of poles calculated at stage 1 may in general contain unstable poles. These are forced to be stable by switching the sign of their real parts, before proceeding with stage 2 .

\subsection{Stabilization procedure}

Fitting the transformer frequency responses with stable poles is a necessary but not sufficient condition for obtaining a stable simulation. In addition, we must require that the transformer model behave as a passive network as seen from its terminals. From circuit theory it is known that passive network behavior implies positive realness of all eigenvalues of the fitted admittance matrix $Y_{f i t}(\omega)$. To ensure positive realness we use the following stabilization procedure, which contains two stages :

\subsubsection{Stabilizing the measured data}

At each frequency point, diagonalize $Y$ into its eigenvalues $\Lambda$ with eigenvectors $T$. All real parts of $\Lambda$ that are negative (due to measurement errors!) are replaced with zero. With the modified eigenvalues and the original eigenvector matrix, retrieve a more realistic $Y$-matrix.

\subsubsection{Stabilizing the rational function approximation}

1. From the SER, calculate $Y_{f i t}(\omega)$ and its eigenvalues $\Lambda(\omega)$ over the frequency range of interest. Find the smallest real. part (most negative) of each eigenvalue within this frequency range, and store in the diagonal matrix $\Lambda_{\min }$. Replace all positive elements in $\Lambda_{\text {min }}$ with 0 . Also, find the highest frequency point $\omega^{*}$ above which all eigenvalues are positive real (if such a point exists).

2. At the lowest frequency point, calculate the eigenvector matrix of $Y_{f i t}$, rotate its columns to minimize the imaginary parts in the least squares sense [4], and discard the imaginary parts. This gives a real transformation matrix, $T_{0}$.

3. Calculate the correction term

$$
Y_{\text {corr }}(\omega)=T_{0} \Lambda_{\min } T_{0}^{-1} \frac{k p}{s-p}=D_{\text {corr }} \frac{k p}{s-p} ; p=-j \omega^{*}
$$

where $k$ is a safety factor, $k \geq 1$.

4. Modify the original SER so that

$$
Y_{\text {fit }}^{\prime}=Y_{\text {fit }}+Y_{\text {corr }}
$$

The above procedure will guarantee positive realness for the eigenvalues of $Y_{f i t}^{\prime}$ if the transformation matrix $T$ for $Y_{f t}$ is frequency independent and real. In the case of the transformer studied in this paper, $T$ was complex and strongly frequency dependent. Despite of this, the stabilization procedure gave the desired positive realness (we used a safety factor $k=1.2$ ).

It should be noted that the eigenvalues calculated in step 1 are accurate eigenvalues, i.e. a complex, frequency dependent 
transformation matrix is used. In this process the correct sequence of eigenvalues is maintained by eliminating any artificial eigenvector switchovers. This is achieved by tracing the direction of the eigenvectors in the eigenspace [5], as was also done in [3].

The intention of the pole in (6) is to "kill" the correction at high frequencies. The rationale is that the eigenvalues of $Y_{f i t}$ are likely to be positive real at high frequencies as the frequency dependent losses in the transformer increase.

The modification of the SER for $Y_{\text {fit }}^{\prime}$ imposed by the correction term is straightforward and so we give only a brief outline of the details. Assume that the pole in (6) is not to be used. In the case of model 1 , the correction is implemented by simply adding $D_{\text {corr }}$ to the $D$-matrix for the transformer SER. If the pole is to be used, then the SER for each column will have its matrices $A, B$ and $C$ modified, as the order increases by one. In the case of model 2, it is more practical to implement the correction term as a SER in parallel with the original SER.

\section{CALCULATED RESULTS}

\subsection{MVA Transformer}

Figure 3 shows the winding arrangement for the transformer studied in this paper. The $Y$-matrix was obtained from measured frequency domain responses in the range $1 \mathrm{kHz}-200 \mathrm{kHz}$. The measured data were supplemented by two additional frequency points at $1 \mathrm{~Hz}$ and $50 \mathrm{~Hz}$, which were calculated from rated data (Appendix B). Figure 3 also defines the numbering of terminals used in subsequent examples.

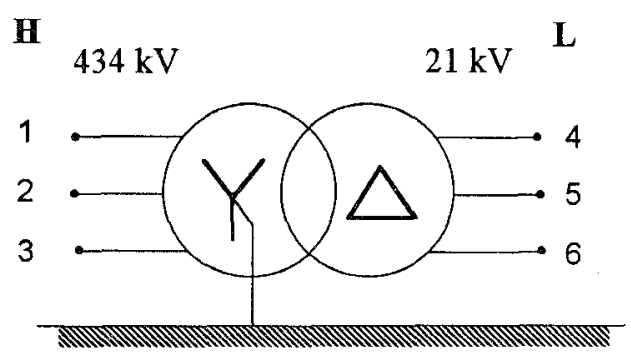

Fig. 3 Transformer layout

\subsection{Phase domain fitting}

In the phase domain approach we fit $Y$ columnwise using the same set of poles for each column. Figures 4 and 5 show the magnitude functions of the 1st and 4th column of $Y$ when approximated by a 12th order SER calculated by the method of vector fitting. When comparing with the measured data, it is seen that the fit is very good. The phase angles were also well fitted, since both the real and imaginary part of $Y$ are considered in the fitting process. It should be noted that $Y$ is accurately fitted also at $50 \mathrm{~Hz}$.

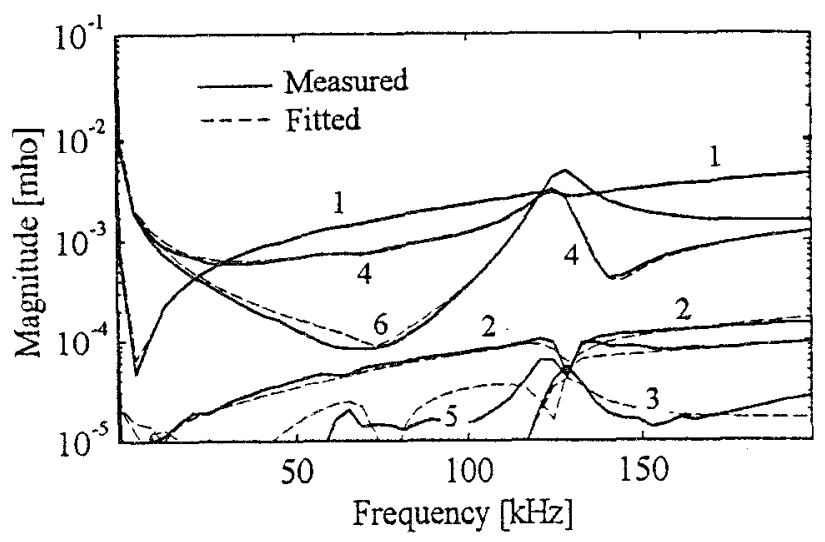

Fig. 4 First column of $Y$ (12th order approximation)

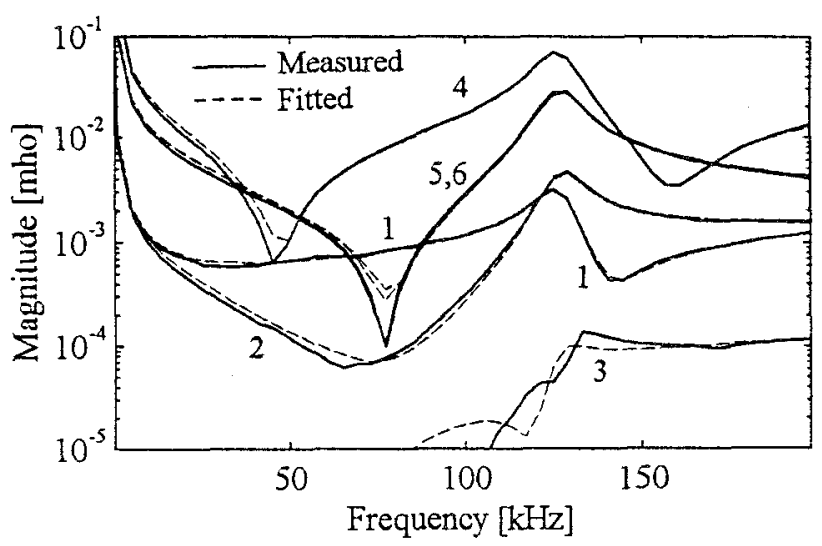

Fig. 5 Fourth column of $Y$ (12th order approximation)

\subsection{Modal domain fitting}

In the modal domain approach we apply modal diagonalization for $3 \times 3$ blocks of $Y$. As described in section 2.1 , we use $\alpha \beta 0$ components for the diagonal blocks, and +-0 components for the off-diagonal blocks. Each eigenvalue is fitted separately as a scalar quantity.

Figure 6 shows the six fitted eigenvalues of blocks $Y_{H H}$ and $Y_{L L}$, where subscripts $H$ and $L$ denote high- and low-voltage windings, respectively. It is seen that the accuracy is very good.

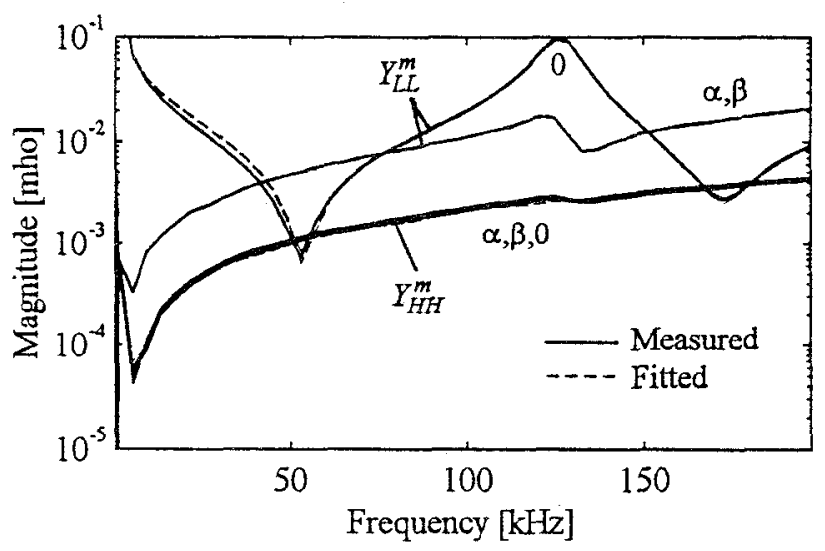

Fig. 6 Eigenvalues of $Y_{H H}$ and $Y_{L L}$ (12th order approximation)

The fitting procedure had to be modified in order to be applicable to the eigenvalues of the off-diagonal blocks. This is explained next.

The phase domain elements of $Y$ are physical quantities, i.e. they have the conjugacy property

$$
Y(s)=Y^{*}\left(s^{*}\right)
$$

The method of vector fitting produces a SER with poles that are either real, or come in complex conjugate pairs. This type of SER automatically satisfies (8), so that it is sufficient to only consider positive frequencies in the fitting process. This will also be the case when fitting eigenvalues produced by a real transformation matrix, as was the case for the diagonal blocks. However, in the case of the off-diagonal blocks, a complex transformation matrix is used ( +-0 components). This entails that two of the eigenvalues (positive and negative sequence) lose the conjugacy property (8). Thus, the eigenvalues of the offdiagonal blocks must be fitted also at negative frequencies using general complex poles. The fitting routine [3] was modified to produce general complex poles by simply removing the constraint of realness for the $C$-matrix. 
Figure 7 shows the eigenvalues of block $Y_{L H}$, when approximated by the modified fitting routine. Again, the accuracy of the fit is very good.

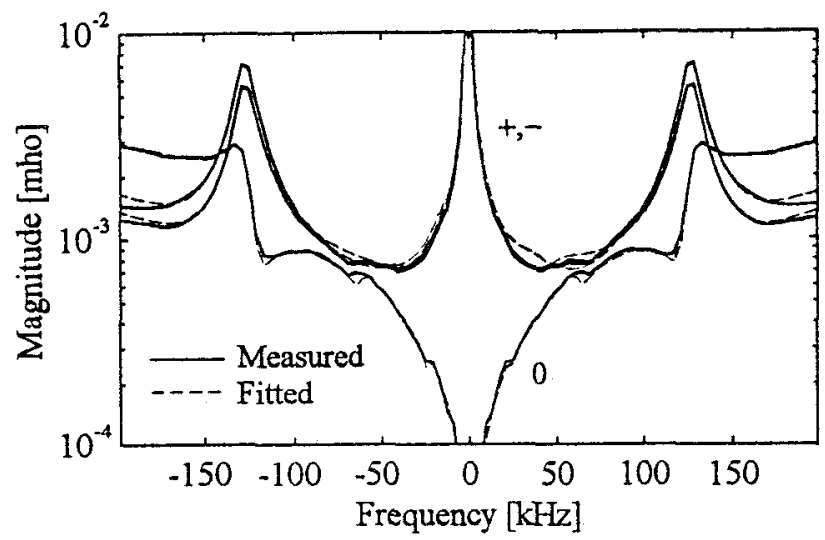

Fig. 7 Eigenvalues of $Y_{L H}$ (12th order approximation)

In order to assess the accuracy of the final transformer model, the corresponding phase domain representation $Y_{f i t}$ was calculated from the fitted eigenvalues and the constant transformation matrices. Columns 1 and 4 of $Y_{f i t}$ are shown in figure 8 and 9 , respectively, together with the measured data. It is seen that where the magnitudes are not very small, the fitting is very good.

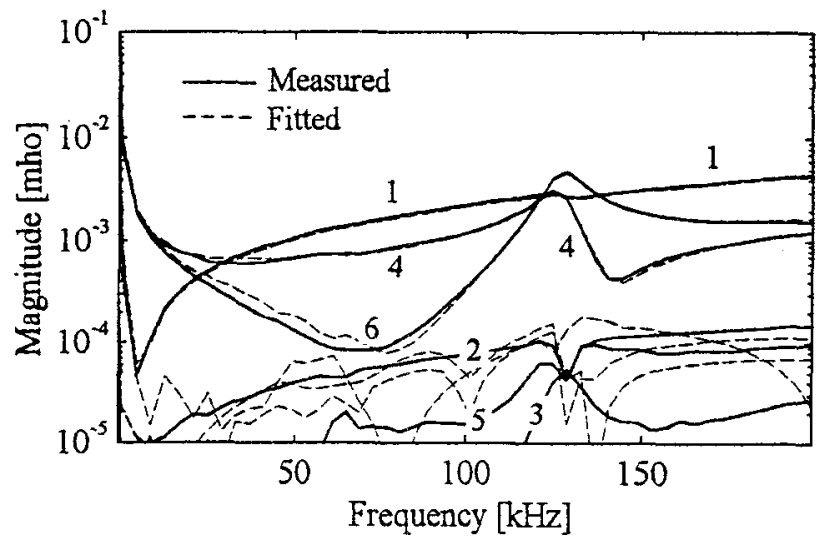

Fig. 8 First column of $Y$ (modal domain approach)

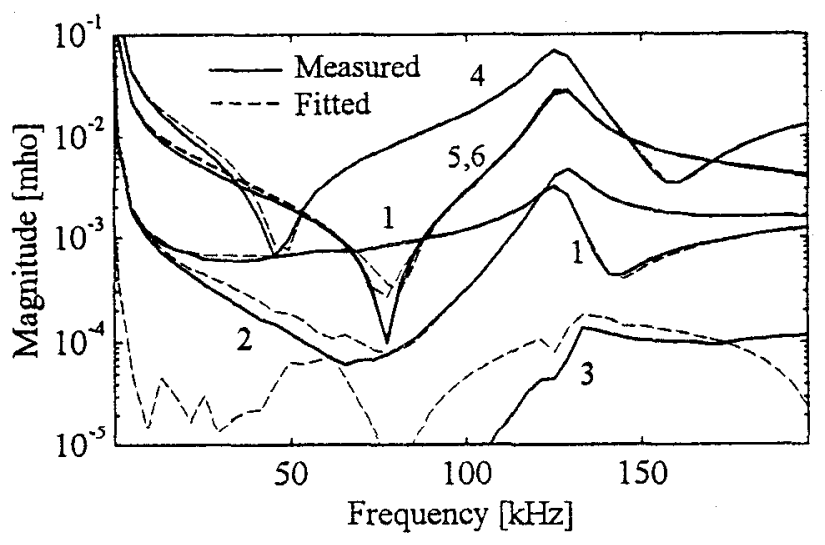

Fig. 9 Fourth column of $Y$ (modal domain approach)
By comparing figures 8 and 9 with the corresponding results using phase domain fitting (figures 4 and 5), we note that the accuracy of the modal domain and phase domain approaches appears to be roughly equal. However, in the case of column 1 (figures 4 and 8) we note that elements which are small in magnitude (2,3 and 5) are fitted much more accurately in the phase domain approach.

\subsection{Application of stabilization procedure}

After finding a SER for the transformer, input-output stability is ensured by the stabilization procedure described in section 2.3 . Figure 10 shows the effect of stage 2 of the stabilization procedure on the real part of the eigenvalues of $Y_{f t}$, when $Y$ was fitted in the phase domain using a 7 th order SER per column.

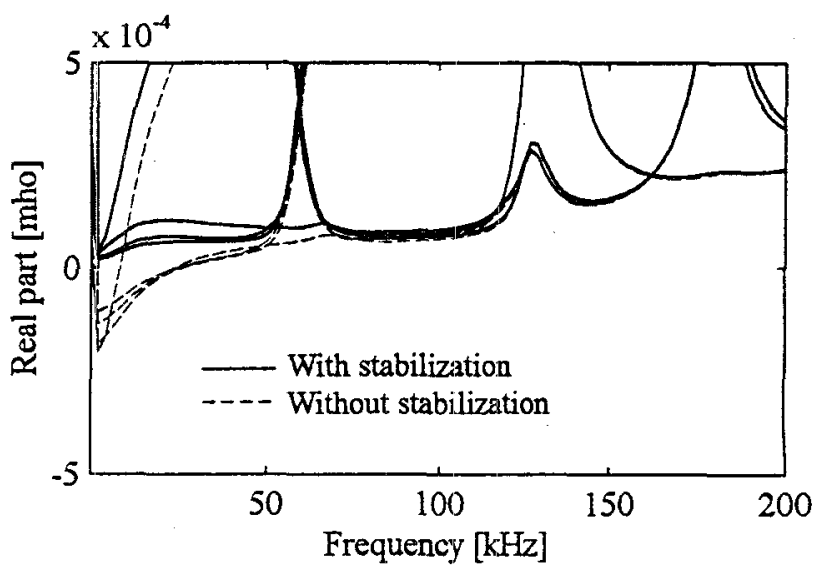

Fig. 10 Effect of stabilization on real part of eigenvalues of $Y$

It is seen that the stabilization has the desired effect of positive realness for the eigenvalues, thus ensuring input-output stability. It should be noted that the correction in figure 10 is very small. The phase domain elements of $Y_{f i t}^{\prime}$ were virtually unchanged by this correction

In the following we show the effect of stabilization on a time domain simulation. Using the same transformer model as in figure 10 , we simulate the voltages at the transformer low-voltage terminals when applying symmetrical step voltages to the highvoltage terminals. At the low-voltage side, $10 \mathrm{nF}$ capacitors are connected to ground, as shown in figure 11.

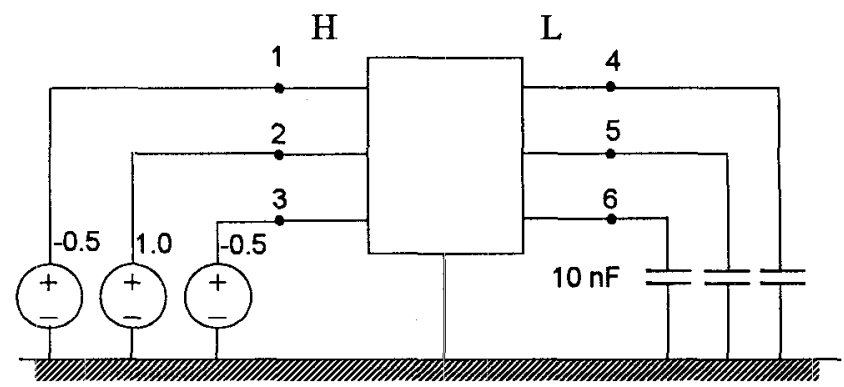

Fig. 11 Energizing transformer with step voltages

The SER for the transformer was converted into a Norton equivalent assuming trapezoidal integration. Norton equivalents were also established for each capacitor, thus allowing a time domain simulation to be run in the same manner as in the EMTP.

Figure 12 shows the simulated voltage at the low-voltage terminals. It is seen that the voltages "blow up" when no stabilization is used, whereas utilization of the stabilization method gives a stable simulation. 


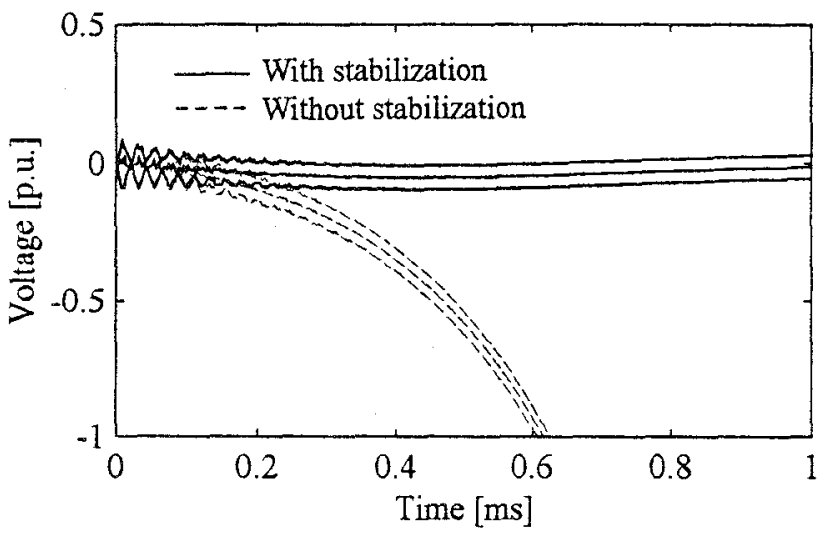

Fig. 12 Simulated voltage at low-voltage terminals

The results in figure 12 were analyzed by calculating a SER for the entire circuit, including both the transformer and the three capacitors. It was found that in the unstabilized case the total SER had two unstable real poles : $p=1346 \mathrm{rad} / \mathrm{s}$ and $p=3421 \mathrm{rad} / \mathrm{s}$. In the stabilized case all poles were stable.

It should be noted that even if a transformer model is inputoutput unstable in some frequency ranges, the simulations may still be stable, depending on the connected network. This is demonstrated in Appendix $\mathrm{C}$ for a simple example.

\subsection{Calculation of pseudo-measurements at high frequencies}

Transformer frequency responses may in practice not be measured sufficiently high up in frequency for the asymptotic behavior to be reached. Therefore, when a measured response is approximated within a confined frequency interval using the method of vector fitting, poles may appear outside the interval considered. These poles contribute to increased accuracy within the frequency interval considered, but may in some instances lead to an undesirable behavior outside the interval.

If the frequency responses of a transformer have been fitted up to a frequency $f_{\max }$, then the time step size should be limited to not less than $\Delta t=1 / f_{\max }$ in order to avoid contributions from spurious high frequency components. However, if on occasion a smaller time step is needed, then the transformer model must have a "reasonable behavior" above $f_{\max }$.

This problem can be solved by calculating additional points above $f_{\max }$ which are included in the fitting process. In this work we calculated two additional points, at $2.5 f_{\max }$ and $5 f_{\max }$, by assuming a linear variation of the magnitude function from the origin through the measured value at $f_{\max }$. The phase angles were set equal to $90^{\circ}$ for the diagonal elements and $-90^{\circ}$ for the off-diagonal elements, corresponding to a capacitive behavior.

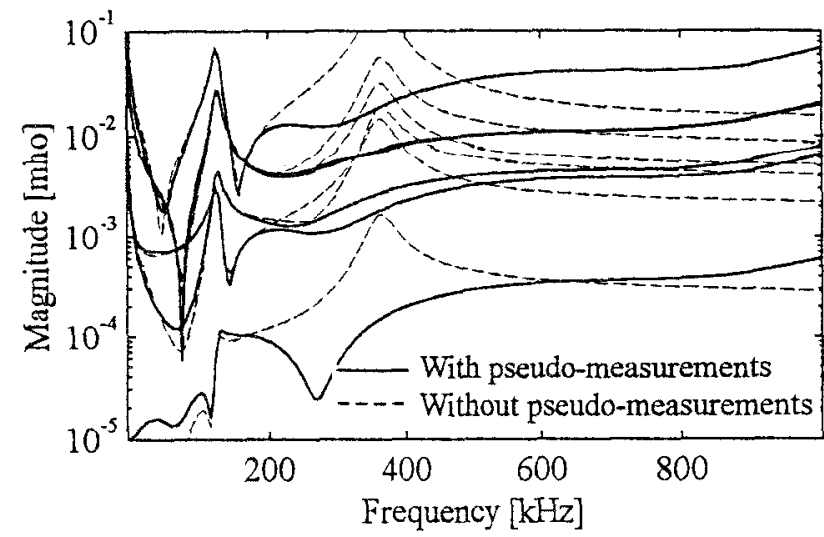

Fig. 13 Inclusion of high frequency pseudo measurements
Figure 13 shows the effect of the two pseudo-measurement on the high frequency behavior of the fitted elements of column 4 . It is seen that a much smoother behavior is achieved.

In principle, an arbitrary number of additional frequency points may be introduced in the fitting process. However, an excessive number of points should be avoided as this will degrade the accuracy of the fit within the measured frequency range, due to the additional constraints.

\section{DISCUSSION}

The calculated results in chapter 3 demonstrated that a frequency dependent transformer model can be formulated by fitting the admittance matrix $Y$ either directly in the phase domain, or by fitting the eigenvalues of blocks of $Y$ assuming a constant transformation matrix.

An advantage of the phase domain approach is that it is more general. For instance, it could be used for finding the SER of a transformer where the measurements included an ungrounded neutral point ( 7 terminals). An additional advantage is that in the phase domain all elements satisfy the conjugacy requirement (8). This avoids the need for general complex poles in the modal domain approach encountered when fitting the positive and negative sequence components of the off-diagonal blocks. It seems therefore that the phase domain approach is both more general and easier to implement in a computer program than the modal domain approach. It should also be noted that usage of general complex poles leads to complex state variables.

In the case of a two-winding transformer, the modal domain approach involves 12 convolutions while the phase domain approach involves 36 convolutions. However, the evaluation of each convolution will be about 2 times faster in the phase domain because all elements in a column share the same set of poles [3]. Therefore, the computational effort appears to be about equal in the two approaches.

\section{CONCLUSIONS}

In this paper we have demonstrated that vector fitting is an efficient methodology for finding a state equation realization (SER) of transformers described by measured frequency responses. Two different models have been developed :

Model 1 is a true phase domain model in which the admittance matrix $Y$ is fitted columnwise. High computational efficiency is achicved since all elements within a column share the same set of poles.

Model 2 is a modal domain approach in which $Y$ is partitioned into $3 \times 3$ blocks, one diagonal block for each winding. The diagonal blocks are diagonalized using $\alpha \beta 0$ components, while +-0 components were used for the off-diagonal blocks because these are cyclic for a star-delta connected transformer. Each eigenvalue is fitted separately.

Application to the measured responses of a 410 MVA twowinding transformer demonstrated that both approaches gave an accurate transformer model using 12 poles per column or 12 poles per eigenvalue. The phase domain model appeared to give a higher accuracy for small elements of $Y$.

The two transformer models are dynamically stable since the poles of the SER are forced to be stable. Input-output stability is ensured by adding a correction term to the SER which forces the eigenvalues of the fitted $Y$-matrix to have positive real parts. It was demonstrated that adding the stabilizing correction term made an unstable simulation stable.

The phase domain approach appears to be more general than the modal domain approach, and is also easier to implement in a computer program. The computational efficiency of the resulting simulation models appear to be about equal. 


\section{ACKNOWLEDGEMENTS}

Financial assistance by the Natural Sciences and Engineering Research Council of Canada is gratefully acknowledged. The authors are indebted to Dr. T. Henriksen (EFI, Trondheim, Norway) and T. Ohnstad (Statnett, Oslo, Norway) for providing the measured frequency responses. The first author wishes to express his gratitude to EFI for granting and financing his leave at the University of Toronto.

\section{REFERENCES}

[1] A. O. Soysal and A. Semlyen, "Practical Transfer Function Estimation and its Application to Transformers", IEEE Trans. PWRD, vol. 8, no. 3, July 1993, pp. 1627-1637.

[2] A. Morched, L. Marti and J. Ottevangers, "A high frequency Transformer Model For the EMTP", IEEE Trans. PWRD, vol. 8, no. 3, July 1993, pp. 1615-1626.

[3] B. Gustavsen and A. Semlyen, "Simulation of Transmission Line Transients Using Vector Fitting and Modal Decomposition", IEEE paper no. PE-347-PWRD-0-01-1997 presented at the IEEE/PES Winter Meeting, New York, 1997.

[4] V. Brandwajn, "Modifications of user's instructions for "MARTI SETUP"", EMTP Newsletter, vol. 3, no. 1, August 1982, pp. 76-80.

[5] L. M. Wedepohl, H. V. Nguyen and G. D. Irwin, "FrequencyDependent Transformation Matrices for Untransposed Transmission Lines using Newton-Raphson Method", IEEE Trans. PWRS, vol. 11, no. 3, August 1996, pp. 1538-1546.

\section{APPENDICES}

\section{A - Accuracy of Constant Transformation Matrices}

In general, the diagonal block matrices $Y_{H H}$ and $Y_{L L}$ of a transformer will have central phase symmetry. In a previous study [2] these matrices were balanced before carrying out the diagonalization. The modified matrix could then be perfectly diagonalized using a constant transformation matrix. In the following we show that better accuracy is achieved if we instead apply $\alpha \beta 0$ transformation to the original matrix, and thereafter discard the off-diagonal matrix elements.

Consider the matrix

$$
A=\left[\begin{array}{ccc}
s & m & m^{\prime} \\
m & s^{\prime} & m \\
m^{\prime} & m & s
\end{array}\right]
$$

Introduce the quantities $\Delta s=s-s^{\prime}, \Delta m=m-m^{\prime}, \varepsilon=\Delta s-\Delta m$.

Diagonalization via $\alpha \beta 0$ transformation yields

$$
B=\left[\begin{array}{lll}
x & 0 & \delta \\
0 & x & 0 \\
\delta & 0 & x
\end{array}\right] ; \delta=\frac{\sqrt{2}}{3} \varepsilon
$$

Backtransformation of the diagonal part of $B$ gives

$$
A^{\prime}=A+\frac{\varepsilon}{9}\left[\begin{array}{ccc}
-2 & 1 & -2 \\
1 & 4 & 1 \\
-2 & 1 & -2
\end{array}\right]
$$

The error in both (A.2) and (A.3) is of order $\varepsilon$, which is less than the error incurred by prebalancing $A$ as in [2] for subsequent diagonalization. (One could define an unbalanced $A$ of (A.1) as "ideal" when $\varepsilon=0$ : then the $\alpha \beta 0$ transformation yields perfect diagonalization even though $A$ is not balanced and its prebalancing would still produce error.)

Similarly, when $A$ has nearly cyclic symmetry, it is more accurate to first apply the +-0 transformation and then discard the residual off-diagonal terms, than using a prebalancing approach.

\section{B - Calculation of Pseudo-Measurements at Low Frequencies}

Assume that the transformer is described by its rated power and rated voltages at the primary and secondary side. In addition, the short circuit impedances $Z_{S C}$ and open circuit admittances $Y_{O C}$ are known for zero-, positive, and negative sequences. From these quantities we can calculate a model at $50 \mathrm{~Hz}$ as follows :

Apply zero-, positive-, and negative sequence voltages to the high voltage side of the transformer with the negative side short circuited to ground. For all of these three tests, calculate the current flowing into all terminals. Repeat the procedure with the voltages applied to the low-voltage side, with the high voltage: terminals connected to ground. This procedure gives 6 voltage vectors of length 6 , and 6 current vectors of length 6 . Stack the voltage and current vectors together in matrices $V$ and $l$, and calculate the admittance matrix at $50 \mathrm{~Hz}$ as $Y_{50}=I \mathrm{~V}^{-1}$.

This method can also be used for calculating $Y$ at an arbitrary frequency $\omega$ by modifying $Z_{S C}$ and $Y_{O C}$ as follows :

$$
\begin{gathered}
Z_{S C}=\operatorname{Re}\left\{Z_{S C_{s 0}}\right\}+j \frac{\omega}{\omega_{50}} \operatorname{Im}\left\{Z_{S C_{S 0}}\right\} \\
Y_{O C}=1 /\left[\operatorname{Re}\left\{Z_{O C_{S 0}}\right\}+j \frac{\omega}{\omega_{50}} \operatorname{Im}\left\{Z_{O C_{50}}\right\}\right]
\end{gathered}
$$

where $Z_{O C_{s 0}}=1 / Y_{O C_{s 0}}$

\section{C - Example Demonstrating Input-Output Stability}

In the following we demonstrate by an example that total positiverealness of the admittance for a component is not necessary for systern stability.

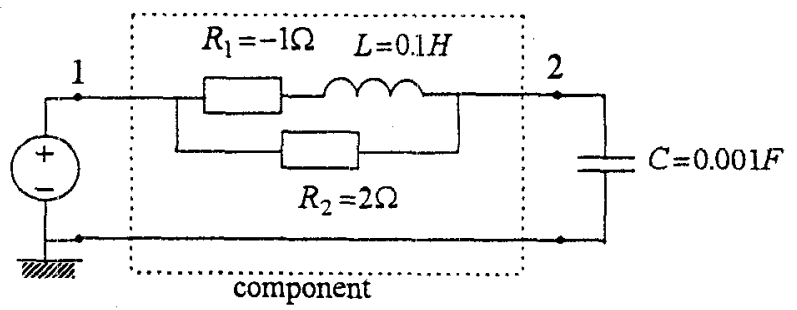

The admittance seen between 1 and 2 is $y=\frac{1}{R_{2}}+\frac{1}{R_{1}+j \omega L}$.

The real part of $y$ is negative for $\omega \in[0,10)$ and positive for $\omega>10$. Thus, $y$ is not positive-real between $\omega=0$ and $\omega=10$.

The eigenvalues for the state equation realization of the complete system were found to be $\lambda_{1,2}=-20 \pm j 10$. Thus, the transient attenuates as $\exp (-20 t)$. This shows that the system is stable despite the fact that the component shown is not globally positive-real.

\section{BIOGRAPHIES}

Bjern Gustavsen (Member, IEEE) was born in 1965 in Harstad, Norway. He received the M.Sc. degree in Electrical Engineering from The Norwegian Institute of Technology in 1989, and the Dr. Ing. Degree in 1993. Since 1994 he has been working at the Norwegian Electric Power Research Institute, mainly in the field of transient studies. He is currently on leave at the Department of Electrical and Computer Engineering. University of Toronto.

Adam Semlyen (Life Fellow, IEEE) was born in 1923 in Rumania where he obtained a Dipl. Ing. degree and his Ph.D. He started his career there with an electric power utility and held academic positions at the Polytechnic Institute of Timisoara. In 1969 he joined the University of Toronto where he is a professor in the Department of Electrical and computer engineering, emeritus since 1988. His research interests include steady state and dynamic analysis as well as computation of electromagnetic transients in power systems. 


\section{Discussion}

Leonardo T. G. Lima, Sandoval Carneiro Jr and Nelson Martins (COPPE/EE/UFRJ - Rio de Janeiro, RJ, Brazil; CEPEL - Rio de Janeiro, RJ, Brazil): The authors have been contributing to the development of models suited to power systems electromagnetic transient analysis with several significant papers, which have been widely used as references in this field.

The paper presents the modeling of transformers with frequency dependent parameters in a state equation realization (SER) of a multiinput multi-output (MIMO) transfer function whose transfer matrix is the admittance matrix $Y$, as shown in equation (1).

A new fitting technique is applied to obtain the rational functions approximations of the elements of $Y$ in a column-wise procedure, which ensures the same poles to all the functions in that column.

A MIMO transfer function can be represented by a state equation realization

$$
\begin{aligned}
\dot{\mathbf{x}} & =\mathbf{A x}+\mathbf{B u} \\
\mathbf{y} & =\mathbf{C x}+\mathbf{D u}
\end{aligned}
$$

where $\mathbf{x}$ is the state vector, $\mathbf{u}$ is the input vector and $\mathbf{y}$ is the output vector with dimensions $p, q$ and $r$, respectively. The transfer matrix can be obtained as

$$
\mathbf{C}(s \mathbf{I}-\mathbf{A})^{-1} \mathbf{B}+\mathbf{D}=\frac{\mathbf{C A d}(s \mathbf{I}-\mathbf{A}) \mathbf{B}+\mathbf{D d e t}(s \mathbf{I}-\mathbf{A})}{\operatorname{det}(s \mathbf{I}-\mathbf{A})}
$$

where the numerator is a matrix with dimensions $r \times q$ and the denominator is a single polynomial in $S$.

This equation shows that all the elements of the transfer matrix share the same poles, i.e., the roots of the polinomial $\operatorname{det}(s \mathbf{I}-\mathbf{A})$ or, equivalently, the eigenvalues of the state matrix $\mathbf{A}$.

Is it possible to extend the vector fitting technique to a matrix fitting technique in which all elements of the matrix $Y$ share the same poles?

A methodology to obtain reduced order models of dynamic systems based on the transfer function dominant poles was proposed in [A], where a SER of a frequency-dependent model of the transmission line was taken into account. The transformer model proposed in the paper can be considered in a similar way to obtain a reduced order models.

Is it easier to fit the rational approximations when considering a larger number of poles ?

Is the methodology applicable to multi-phase transformers and autotransformers?

The comments of the authors on these topics will be appreciated.

\section{REFERENCE}

\section{[A] Leonardo T. G. Lima, Nelson Martins \& Sandoval Carneiro Jr. - "Dynamic} Equivalents for Electromagnetic Transient Analysis Including Frequency-Dependent Transmission Line Parameters", Proceedings of IPST'97 International Conference on Power Systems Transients, Seattle, USA, $22^{\text {cond }}-26^{\text {th }}$ June 1997, pp. 131-136.

Manuscript received February 25, 1998.

Taku Noda (Central Research Institute of Electric Power Industry - CRIEPI, Tokyo, Japan) and Akihiro Ametani (Doshisha University, Kyoto, Japan): We would like to congratulate the authors for developing the new method of frequency-dependent transformer modeling using the vector fitting.

In ref. [A], Wilcox applied the modal theory to transformer modeling based on traveling waves along windings as shown in Fig. A (a). Fig. A (b) shows the equivalent transmission-line representation of the windings, where the modal theory can easily be applied in the same manner as a conventional transmission line. In comparison between the proposed model and Wilcox's regarding computational efficiency and numerical stability, the comments of the authors would be highly appreciated.

In section 3.1, the frequency responses of $\boldsymbol{Y}$ are measured from $1 \mathrm{kHz}$ to $200 \mathrm{kHz}$. The frequency range seems to be too small for accurate simulations. From our experience, the low-frequency behavior of $Y$ is especially necessary to be taken into account, in order to avoid dc bias errors at a wave tail.

Only Fig. 12 shows a result of transients calculation. It would be appreciated if other practical transients calculation results are presented. 
[A] D.J. Wilcox, "Theory of transformer modelling using modal analysis," IEE Proc. Pt. C, Vol. 138, No. 2, 1991.

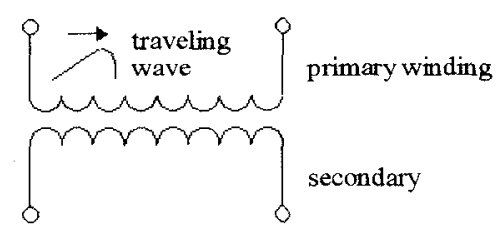

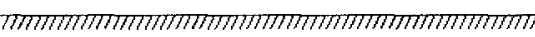

(a) transformer windings

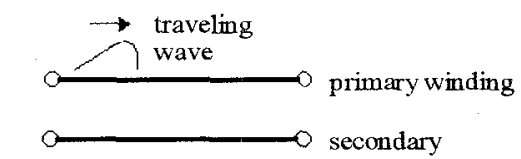

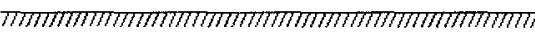

(b) equivalent transmission line

Fig. A Wilcox's transformer model

Bjørn Gustavsen and Adam Semlyen: We wish to thank the discussers for their interesting questions and remarks which will be addressed in the following.

\section{Mr. Lima, Professor Carneiro, and Dr. Martins:}

The idea of a state equation realization (SER) in the classical form (I) suggested in the discussion (see also reference [A]) is not only of theoretical interest but also of significant practical value [1]. Essential to the idea of pole sharing by all elements of the resultant transfer function matrix $\mathbf{H}$ is the concept that all state variables $\mathbf{x}$ are related to a common "kernel" of the transformer model, where all dynamic phenomena take place, and the inputs $\mathbf{u}$ and outputs $\mathbf{y}$ are related to these only through the respective constant matrices, $\mathbf{B}$ and $\mathbf{C}$. If however the inputs are taken sequentially, one by one, which corresponds to vector fitting, then some of the state variables will be affected repeatedly, again and again, so that the sequential column-wise realization of $\mathbf{H}$ and of $\mathbf{B}$ will lead to a non-minimal model. Thus, clearly, an optimal fitting of form (I) is of lower dynamic order than other alternatives.

The practical difficulty is of course devising a successful nonlinear least squares (NLLS) process for the efficient identification of model (I). We have obtained a nearly optimal realization of form (I) in the case of transmission lines by using a procedure we called polar decomposition [B]. It consists in determining the poles contained in $\mathbf{A}$ using the eigenvalues of $\mathbf{H}$ and then identifying $\mathbf{B}$ and $\mathbf{C}$ by a sequence of linear solutions, "BC-iterations", with $\mathbf{C}$ and $\mathbf{B}$ alternatively taken as fixed and known. The method could be applied to transformers and even used to obtain initial values in a full NLLS computation of the SER (I).

The advantage of vector fitting is that it solves the nonlinear problem by using only basic linear solution techniques of numerical linear algebra. This is achieved by: $(i)$ splitting $\mathbf{B}$ into its columns (which leads to fitting each vector of $\mathbf{H}$ separately) and normalizing each column $\mathbf{b}$ to $1 \mathrm{~s}$ (such normalization to fixed numbers would not be possible for the complete B matrix); and (ii) choosing poles with an appropriate distribution. The remaining problem of calculating $\mathbf{C}$ and of scaling are then linear. This results in vector fitting being fast, accurate, very robust and reliable, and applicable to the most difficult systems, without however producing a minimal SER.

The vector fitting technique described in the paper could in fact be easily applied for obtaining a model where all elements in a matrix $\mathbf{H}$ share the same poles. This is done simply by stacking all elements of $\mathbf{H}$ into a single vector, and applying vector fitting to the vector. However, this will not lead to computational savings in the time domain simulations because poles of different columns need separate realizations since the state variables associated with them are excited by different inputs.

The accuracy of the rational approximation of the frequency responses increases with increasing order of the fitting. In the case of many peaks in the transfer function the number of poles needed could become quite large. The same is true when modeling external dynamic system equivalents. The large number of poles can be attributed to successive wave reflections. Therefore the methodology of the discussers' reference [A] of dealing with this problem is of particular interest.

The methodology described in the paper is quite general-it is applicable to both multiwinding transformers (e.g. the two winding transformer in the paper) and to autotransformers. The only limitation is that the frequency responses should not be influenced by nonlinear effects.

\section{Dr. Noda and Professor Ametani:}

The transformer model developed in reference [A] of the discussion is based on discretizing the windings into sections, and each section is modeled using physically realizable components only (positive $R$ 's, $L$ 's, $C$ 's). Therefore this model will always be stable in a simulation (due to stable poles and passive circuit behavior). It is difficult to comment on the efficiency of the model, as it is not clear how the order of the model relates to its accuracy. The model could be very useful for assessing winding stresses, but it requires detailed information about the transformer, which is normally proprietary to the manufacturer. We therefore think that for calculation of general power system transients, a practical transformer model should be based on measured characteristics at its terminals.

The frequency responses were measured from $1 \mathrm{kHz}$ to 200 $\mathrm{kHz}$. For frequencies below this range we have calculated additional frequency points as shown in Appendix B of the paper. Based on the full set of frequency domain data, vector fitting gave a state equation realization that, by itself and the resulting time domain simulations, is as good as the available data. Any number of transient simulations can then be routinely performed as illustrated in figure 12

We take this opportunity to emphasize a few additional details:

We note that although the correction of the eigenvalues at low frequencies (figure 10) guarantees a stable simulation, the low frequency transients are not obtained accurately. This is demonstrated in figure A below, which shows the effect of using twice the correction used in figures 10 and 12 . In general, it is therefore best to try avoiding the stability problem altogether, by measuring accurately, and by fitting 
accurately. Still, the realization obtained may be unstable outside the frequency range used for fitting. Therefore, the importance of assuring the stability of the model cannot be overemphasized and consequently the fitting should include checking procedures for input-otput stability (i.e. passive circuit behavior). While stability has to be assured, the checking should not be excessively conservative since that would lead to reduced accuracy.

The view has sometimes been expressed that the positive-real requirement should be used for the real part $G$ of the admittance matrix $Y=G+j B$. This criterion is overly conservative since it may indicate instability, as we show below, while the $Y$ matrix is still positive real.

The requirement of positive-realness is only indirectly related to stability since it is not linked to the dynamics of the device being examined. It simply establishes that a component never behaves as a negative resistance thereby adversely affecting the stability of the system of which it is a part. The requirement is purely algebraic and can be justified by energy considerations. The component is assumed to be passive. We have then:

$$
i=Y v=(G+j B) v
$$

where $i, v$ are complex vectors; $i$ is into the circuit. Since $v$ can be viewed as a linear combination of the (complex) eigenvectors of $Y$, we may focus on a $v$ that is equal to an eigenvector associated to an eigenvalue $\lambda$ of $Y$. Then (a) becomes

$$
i=\lambda v
$$

The total (real) power into the circuit is

$$
P=\operatorname{Re}\left(v^{*} i\right)=\operatorname{Re}\left(\lambda\|v\|^{2}\right)
$$

Thus $P$ is positive if and only if for all eigenvalues $\lambda$ and for all frequencies

$$
\operatorname{Re}(\lambda)>0
$$

At $\omega=0$ (a) reduces to

$$
i=G v
$$

and one could use an argument similar to that made above for a positive-real requirement (d), this time for the eigenvalues of the matrix $G$. However, since $G$ is real and symmetrical, its eigenvectors are real and therefore do not span the complex vector space we need for representing at all frequencies the complex vectors $i$ and $v$. Therefore, the positive-real requirement (d) is valid only for $Y$ and not for $G$. In fact, when (d) is satisfied for $Y$ (the component is input-output stable), it may not be satisfied for $G$ (but if it is satisfied for $G$ it will also be for $Y$ ). For example, if $G=\left[\begin{array}{cc}1 & -2 \\ -2 & 3\end{array}\right]$ and $B=\left[\begin{array}{cc}24 & -18 \\ -18 & 22\end{array}\right]$, the real parts of the eigenvalues of $Y$ are both positive $(0.0549$ and 3.9451), while one of the eigenvalues of $G$ is negative (they are: - 0.2361 and 4.2361). Thus, assessing input-output stability using the positive-real criterion for $G$ is safe but unduly conservative: the $Y$ matrix should be used instead.

Since the time this paper was written, the method of Vector Fitting has been substantially improved: an ill-conditioning problem has been removed by allowing complex starting poles (particularly useful for fitting data with many peaks in the frequency response), and an unknown constant term as well as an unknown proportional term have been introduced in the rational approximation (5). A paper describing the new method has been submitted to IEEE. As an example, figure B shows the 4 th column of $Y$ fitted with 30 poles. It is seen that high accuracy has been achieved for all elements, including the small one. It should be noted that the fitting was still very good when using only 8 poles (but the approximation of the smallest element became poor at low frequencies).

[A] A.O. Soysal and A. Semlyen, "State Equation Approximation of Transfer Matrices and its Application to the Phase Domain Calculation of Electromagnetic Transients", IEEE Trans. PWRS, vol. 9, no. 1, February 1994, pp. 420-428.

[B] B. Gustavsen and A. Semlyen, "Calculation of Transmission Line Transients Using Polar Decomposition", IEEE paper no. PE-755PWRD-0-04-1997, presented at the 1997 IEEE/PES Summer Meeting in Berlin, Germany.

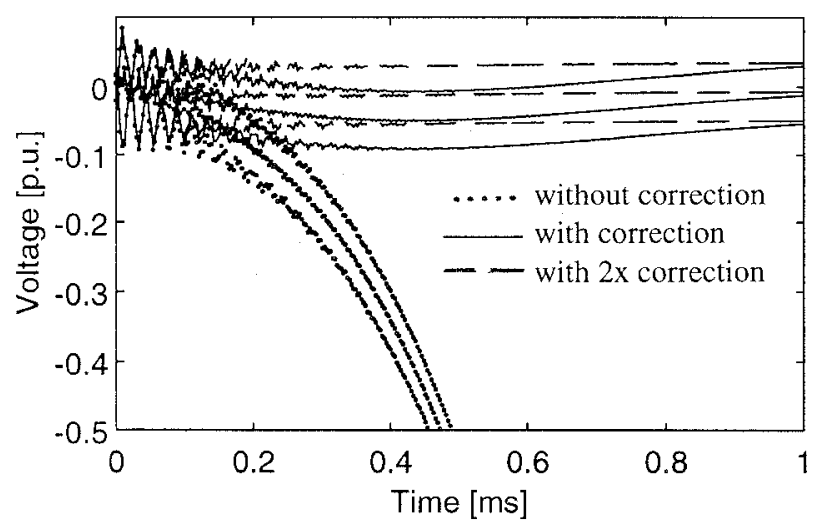

Fig. A Effect of using twice the eigenvalue correction
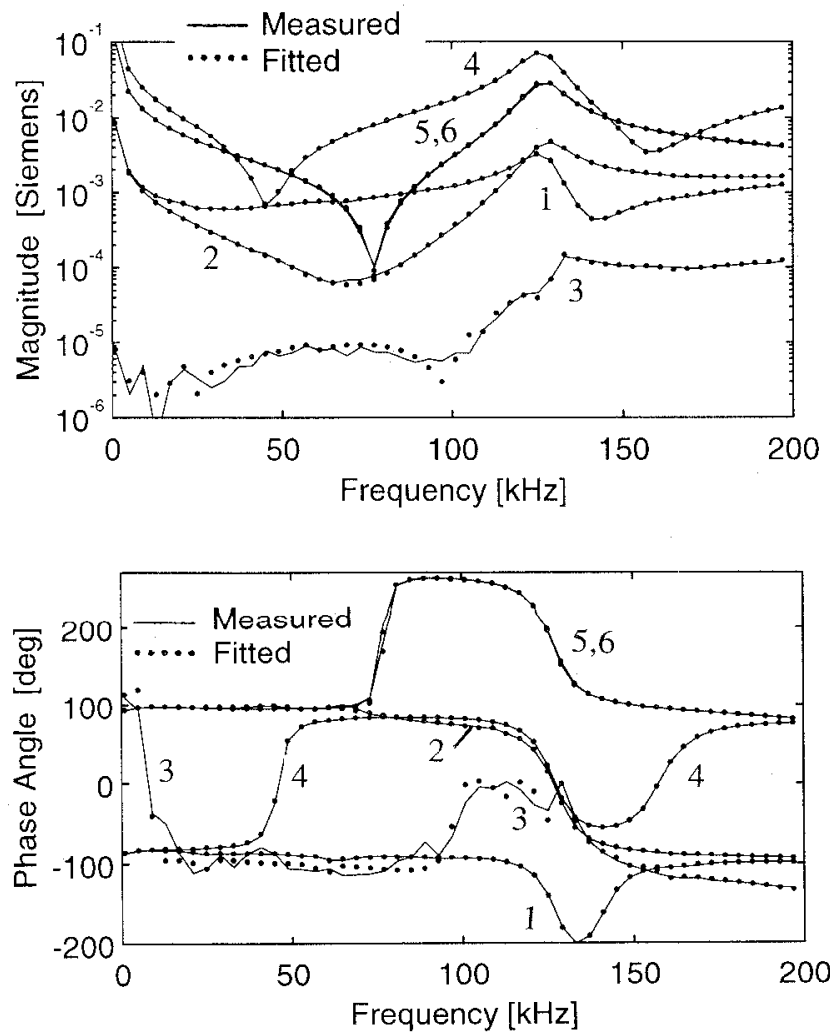

Fig. B Approximation of 4th column of $Y$ by new version of vector fitting (30th order approximation) 